\title{
The preparation and administration of intravenous drugs before and after protocol implementation
}

\author{
Mirjam Tromp • Stephanie Natsch • \\ Theo van Achterberg
}

Received: 14 February 2008/ Accepted: 9 November 2008/Published online: 3 December 2008

(C) The Author(s) 2008. This article is published with open access at Springerlink.com

\begin{abstract}
Objectives This paper reports on a pilot study examining the incidence of nurses' errors in preparation and administration of intravenous drugs. Furthermore, the study aimed to evaluate the short-term effects of implementation of a new protocol for preparation and administration of intravenous drugs. Setting Two nursing departments of internal medicine at a 953 beds University Medical Centre in The Netherlands. Methods By means of a prospective, quasi-experimental design, nurses were observed during the process of preparation and administration of intravenous drugs. Observation was performed before and after the implementation of a new protocol. Seventy-two nurses at two nursing departments were observed during the study. Main outcome measure A mean pre-test and post-test quality score at two departments of internal medicine. Results At baseline, average quality scores for nurses at the two departments were 64 (intervention ward) and 67 (control ward) on a 0-100 quality scale. The pre-test quality scores were not statistically significant for the two nursing
\end{abstract}

\footnotetext{
M. Tromp

Department of Internal Medicine and Nijmegen Institute for Infection, Inflammation and Immunity (N4i), Radboud University Nijmegen Medical Centre, Nijmegen,

The Netherlands

S. Natsch $(\bowtie)$

Department of Clinical Pharmacy and Nijmegen Institute for Infection, Inflammation and Immunity (N4i), Radboud University Nijmegen Medical Centre, Internal Postal Code 864, P.O. box 9101, Nijmegen $6500 \mathrm{HB}$, The Netherlands

e-mail: s.natsch@akf.umcn.nl

T. van Achterberg

Scientific Institute for Quality of Healthcare Nursing Science Section, Radboud University Nijmegen Medical Centre, Nijmegen, The Netherlands
}

wards $(T=1.36, \quad$ df $=55, \quad P=0.18)$. After the implementation of the new protocol, nurses at the intervention ward scored better (72) than nurses at the control ward (69). The mean score at the intervention ward was significantly higher than the score in nurses of the control ward $(T=-2.20$, df $=53, P=0.04)$. Conclusions The number of errors in the preparation and administration of intravenous drugs is high. This study shows that implementing a protocol for the preparation and administration of these drugs can reduce the number of errors.

Keywords Drug administration routes · Infusions · Injections · Intravenous administration · Netherlands . Nursing research · Parenteral · Practice guideline . Quality assurance

\section{Impact of findings on practice}

- Errors in preparation and administration of intravenous drugs occur frequently and can cause irreparable damage to patients.

- Errors in the preparation and administration of intravenous drugs decrease significantly after implementation of a protocol, leading to improved quality of care and patient safety.

\section{Introduction}

Patient safety is an important issue in health care today. For the USA it was estimated that more than a million injuries and 44,000-98,000 deaths per year are related to suboptimal care or mistakes made by health care workers [1]. Yet, 
hospitalized patients are, as a rule, vulnerable and they assume that health care will improve, rather than compromise, their health. Therefore, improving patient safety is an important area for practice improvement as well as research, and it covers a large variety of topics such as preoperative cardiac events, surgical items left in patients, pressure ulcers, hospital acquired infections, falls and fall injuries and delirium [2].

Adverse drug events are among the most common types of health care errors [3-7]. In a study by Bates et al. $1 \%$ of all adverse drug events were fatal, $12 \%$ were life threatening, $30 \%$ were serious and $57 \%$ were significant [8]. In the same study, $40 \%$ of all events were due to mistakes in the process of administering drugs. The risk involved seems especially high in the more complicated medication administration processes, such as in the administration of intravenous drugs.

The preparation and administration of intravenous drugs is a series of complicated technical skills. The consequences of medication preparation and administration errors can be anything from relatively harmless to lethal. Single site studies in the UK and USA confirm that nurses make mistakes in preparing and administering intravenous drugs in $13-84 \%$ of all cases $[9,10]$. These studies used different definitions for 'errors' in preparing and administering intravenous drugs, thus making comparisons difficult. The study of Taxis and Barber describes the different stages in the process in which errors occur, as well as the clinical importance of these errors [11]. In this study, one or more errors occurred in $49 \%$ of all cases of the preparation and administration of intravenous drug doses. Preparation errors occurred in 7\%, administration errors occurred in $36 \%$ and both types of errors were found in $6 \%$ of all cases. $1 \%$ of these errors were potentially severe, $29 \%$ were potentially moderately harmful and $19 \%$ were considered to be minor errors.

Examples of errors observed in practice and described in studies are:

- administering a bolus dose too quickly;

- not inspecting the medicine for expiration date;

- not double-checking the prescribed drug, doses and corresponding patient by a colleague;

- not observing hygiene regulations [11-13].

In conclusion, the evidence of quality and errors in the preparation and administration of intravenous drugs is rather scarce, but points towards a potentially problematic quality of care issues. The study by Cousins et al. compares the intravenous therapy protocols for hospitals in the UK, Germany and France [12]. The study by Anselmi et al. verified the frequency of errors in the preparation and administration of intravenous drugs in three Brazilian hospitals in the States of Bahia [14]. In all hospitals, various difficulties in the developing and maintaining of intravenous therapy protocols were identified. However, attempts to improve the quality of the process of preparing and administering intravenous drugs are minimal described in the international literature $[15,16]$. Yet, the quality of this process is of vital importance in providing safe patient care and attempts at quality improvement deserve to be explored.

These clinical observations, and the motivation to guarantee patient safety, were the reasons to examine the quality and the effects of an attempt towards quality improvement of the preparation and administration of intravenous drugs in a Dutch academic hospital.

\section{Aims of the study}

The aims of the study were:

- To evaluate the quality of the preparation and administration of intravenous drugs as performed by nurses in general medicine hospital wards;

- To evaluate the effects of the implementation of a protocol on the quality of the preparation and administration of intravenous drugs.

\section{Methods}

The study employed a quantitative, prospective, quasiexperimental design. The researcher observed the performance of nurses during the process of preparation and administration of intravenous drugs at two nursing departments of internal medicine at a 953 beds University Medical Centre in The Netherlands.

The study included data collection at baseline (pre-test: 8 weeks), an intervention period while the new protocol was implemented in one of the two wards ( 2 weeks), and a post-implementation test (post-test: 8 weeks). Nurses' performance in the preparation and administration of drugs was the main focus of the study.

Inclusion

Nurses were eligible for the study if:

- They were Registered Nurses at the departments of internal medicine;

- They were fully qualified for the preparation and administration of intravenous drugs;

- They declared themselves competent in the preparation and administration of intravenous drugs;

- They gave permission for observation by the researcher. 
As nurses were also observed during the administration of drugs, patient encounters were part of the observations.

Therefore, an additional inclusion criterion for the observations was:

- Patients receiving drugs should (be able to) give informed consent for the observations by the researcher.

\section{Protocol and observation list}

A multidisciplinary team including two researchers, a hospital pharmacist, a nurse practitioner, a ward nurse, an infection control practitioner and an occupational hygienist developed the new protocol, using the knowledge of experts and the evidence from the scarce literature [17-19]. An observation list was directly derived from this protocol. The observation list is a specification of the protocol and consisted of 47 variables (Appendix). The variables represent the steps a nurse has to take in the preparation (30) and administration (17) of intravenous drugs. Some of the steps during the process of preparation and administration had to be repeated more than once. All 47 aspects on the list were observed during all occasions where nurses prepared and administered intravenous drugs.

With the observation list the option "yes", "no", "not applicable" or "missing" could be checked. When the observer was not sure of his observation then one or more aspects were scored as "missing". If the operation of a step was not complete, for example, the nurse did not wait before the disinfection solution to dry, that item was scored as a "no". All variables were given equal weights. Scores on the items were combined in an overall quality score to represent the number of items that were correctly performed. This overall quality score was expressed on a $0-100$ quality scale. When all 47 aspects were performed correctly, the quality score for preparation and administration was 100. The scores "not applicable" and "missing" were not used in calculating the overall quality score.

In addition, more general data such as date, department, time of prescription and time of administration were collected. Furthermore, characteristics of the administration process (bolus dose injections, intermittent infusion or continuous infusion) were always registered.

\section{Observation}

At both the pre- and post-test phases, nurses were observed by a single observer for a maximum number of four times, in order to include as many different nurses as possible. During the process of preparing and administering intravenous drugs, nurses were observed by using the observation list. Nurses were aware of the observation but unaware of its true purpose [20,21].

The names of the nurses, the number of observation by the individual nurse and the phase of the study were registered.

Observation took place on different days of the week, at different times of the day and night, and at both hospital wards. The observer was present during a preset series of shifts, to represent the variation of working hours in nursing practice.

It was determined beforehand when intervention would be necessary. The project team decided that in case of preparation of the wrong drug, preparation of the wrong dose, preparation with the wrong diluent, administration using the wrong route and administration to the wrong patient, the researcher should not only score the errors, but also intervene by talking to the nurse about these potentially harmful errors.

\section{Intervention}

The intervention was the implementation of the protocol regarding the preparation and administration of intravenous drugs at one of the departments, the intervention department. Implementation took place, during a one-time 45 min department meeting by means of a presentation of the pre-test results and a $15 \mathrm{~min}$ film with and without the presentation of errors in the preparation and administration of intravenous drugs. In addition, questions were answered and current practices versus the new protocol were discussed. For instance, one of the items discussed was the disinfecting of the hands in combination with the wearing of gloves. Nurses thought that when they wore gloves they do not have to disinfect their hands.

Following the presentation, the new protocol was available to all the nurses at the ward. To give all the nurses the opportunity to study the new protocol, the post-test took place 2 weeks after the introduction.

Statistical analysis

At the item level, differences between the two groups and measurements were analyzed using cross table and chisquare tests. The standard deviations for the two groups were not statistically different. Overall quality scores were checked for normal distributions and satisfied this condition. For both pre- and post-test scores, potential differences in nurses' level quality score between the two departments were analyzed using the independent samples T-test for statistically significance $(P \leq 0.05)$. As nurses could be observed several times, clustering of data at the level of nurses was present in this study. Therefore, the mean quality scores of nurses over 1-4 observations were 
used in the analyses of the effects of the protocol implementation. In addition, we explored the relevance of the type of administration route for quality scores using a One Way ANOVA technique $(P \leq 0.05)$.

Ethical considerations

Approval from the local Medical Ethics Committee was obtained.

\section{Results}

At the pre-and post-test, 132 observations were performed; 66 observations at each ward. Seventy-two nurses were observed in the study.

In $56 \%$ of the cases, the nurses were observed at the pretest as well as the post-test (45\% control department, $66 \%$ intervention department). The demographic data are summarized in Table 1. There were no significant differences in any of the demographic data for the two departments.

All of the included nurses gave permission for observation by the observer.

During the study, intervening at the intervention department was necessary on two occasions: once due to preparation of the wrong dose during the pre-test and once due to preparation of the wrong drug during the post-test. In these cases, the nurses corrected their mistakes after the observer had intervened. The mistakes were registered as errors. Intervening at the control department was not necessary.

Nurses working at the control department had a mean quality score of 67 during the pre-test. Nurses from the intervention department scored 64 at this point in time. Pretest scores were not significantly different for the two nursing wards $(T=1.36, \mathrm{df}=55, P=0.18)$. In addition, within each department there was no significant difference in quality scores for the administration by bolus dose injections, intermittent infusion and continuous infusion $(\alpha \leq 0.05)$. The mean overall quality score during the pretest was 66 for bolus dose injections $(n=38), 65$ for intermittent infusion $(n=76)$ and 66 for continuous infusion $(n=18)$. The quality score for bolus dose injections differed for the two departments $(T=2.63, \mathrm{df}=36$, $P=0.01)$. At the control department the quality score for this administration route was 71 , while at the intervention department the score was only 64 . For administration by intermittent infusion or continuous infusion, no significant differences between the wards were found.

Fifteen variables had pre-test quality scores $>90 \%$ at both departments (Appendix). These variables referred to the preparation of the correct drug, correct doses, making a calculation for the solution to be made, using the right diluent, using the prescribed quantity of this diluent, using the prescribed method of preparation, applying the prescribed administration route, keeping syringe, needles and/ or transfer needles sterile, being sure that the medication completely dissolves, having - at the final stage- the prescribed dose of the drug to be administered, administration by the nurse who prepared the drug, administrating the drug before the end of sell-by after preparation and administration of the drug to the right patient.

Eight variables had pre-test scores less than $20 \%$ at both departments (Appendix). The scores referred to checking the expiration date, inviting colleagues to double-check the prescription, inspecting the diluent for expiration date, hand washing prior to preparation, using disinfectant gauze to break/open the ampoule, mixing the acquired infusion solution by wheeling, inspecting the solution for clarity and inspecting the intravenous catheter for phlebitis or extravasation prior to administration.

The mean quality scores of nurses at the control and intervention department are summarized in Table 2. At post-test, nurses at the intervention department scored significantly better (72) than nurses at the control department (69) $(T=-2.20, \mathrm{df}=53, P=0.04)$. At the intervention department, significant improvement on 8 criteria $(P<0.01)$ was found (Table 3$)$.
Table 1 Pre-test $(n=57)$ and post-test $(n=56)$ demographic data for all nurses at the two departments

\begin{tabular}{|c|c|c|c|c|c|}
\hline \multicolumn{3}{|l|}{ Pre-test } & \multicolumn{3}{|l|}{ Post-test } \\
\hline & Control & Intervention & & Control & Intervention \\
\hline Number of nurses & 26 & 31 & Number of nurses & 29 & 27 \\
\hline Male & 5 & 5 & Male & 6 & 3 \\
\hline Female & 21 & 26 & Female & 23 & 24 \\
\hline Mean age & 37 & 37 & Mean age & 36 & 35 \\
\hline Minimum & 23 & 23 & Minimum & 23 & 23 \\
\hline Maximum & 58 & 58 & Maximum & 56 & 57 \\
\hline Mean years of experience & 9 & 9 & Mean years of experience & 8 & 7 \\
\hline Minimum & 0 & 1 & Minimum & 0 & 1 \\
\hline Maximum & 32 & 30 & Maximum & 25 & 30 \\
\hline
\end{tabular}


Table 2 The quality of preparation and administration of intravenous drugs at pre- and post-test observations (0-100 quality range)

\begin{tabular}{clllll}
\hline & \multicolumn{2}{l}{ Control department } & & \multicolumn{2}{l}{ Intervention department } \\
\cline { 2 - 3 } \cline { 5 - 6 } & $\begin{array}{l}\text { Pre-test } \\
{[n]}\end{array}$ & $\begin{array}{l}\text { Post-test } \\
{[n]}\end{array}$ & & $\begin{array}{l}\text { Pre-test } \\
{[n]}\end{array}$ & $\begin{array}{l}\text { Post-test } \\
{[n]}\end{array}$ \\
\hline $\begin{array}{c}\text { Mean quality score } \\
\text { of all nurses }\end{array}$ & $67[26]$ & $69[31]$ & $64[29]$ & $72[27]$ \\
$\begin{array}{c}\text { Mean quality score } \\
\text { of nurses who were } \\
\text { observed at both } \\
\text { pre- and post-test }\end{array}$ & $67[17]$ & $69[23]$ & $65[17]$ & $72[23]$ \\
\hline
\end{tabular}

In the post-test, $37 \%$ of the 27 observed nurses at the intervention ward, were involved the new training. We checked for the effects of 'exposure to the intervention' by comparing the scores for nurses who did or did not attend the protocol introduction meeting. There was a significant difference between the mean score of nurses at the intervention department who were observed during the post-test and who had participated in the department meeting $(n=10$, score $=75)$ as compared to those who were observed during the post-test but who had not attended the meeting $\quad(n=17, \quad$ score $=70) \quad(T=2.27, \quad \mathrm{df}=64$, $P=0.03)$.

\section{Discussion}

This study describes the effect of the implementation of a protocol on the quality of care of preparation and administration of intravenous drugs by nurses. After the implementation of the protocol, we observed a modest significant improvement at the intervention department, whereas no quality improvement was found at the control ward. There are a few studies which also described the effect of an educational intervention on the quality of care of preparation and administration of intravenous drugs, such as using an interactive CD-ROM program [15] and introduction of dedicated medication nurses [16].
However, most studies described errors in the process of preparation and administration of intravenous drugs and made recommendations about how to minimize the errors $[9,10,22]$.

Medication errors range from those that have little or no impact on the patient to those with very serious consequences. In this study, we did not assess the clinical importance of intravenous drug errors. It is difficult to identify the clinical effects of errors because of the delay between the occurrence and the identification of errors and the outcome in one patient may not reflect the likely outcomes in groups of patients as a whole [23].

Potentially severe errors such as preparation of the wrong doses, choosing wrong solvents and administration of bolus doses within insufficient time, are examples of intravenous drug errors that could be severe. They occurred less frequently than previously reported [13].

There was a significant difference between the mean scores of nurses at the intervention department who had participated in the department meeting as compared to those who had not attended the meeting. Despite that, the post-test scores for the nurses who attended the education session are similar to the whole post-test intervention ward group. Perhaps the availability of, and giving attention to the new protocol to all nurses at the ward was equal to, or more effective than the education session itself. Alternatively, carry-over effect from nurses who attended the department meeting to nurses who were not present could explain the lack of the difference between the two subgroups.

Possibly the short period of implementation and the minimum investment caused the modest improvement in quality of care. To accomplish a major change in the attitude and the competence of nurses, a more multi-faceted strategy might be needed. Only $37 \%$ of the 27 observed nurses at the intervention ward, were involved the new training. Another strategy for better up take should be to have more nurses attend the training. In addition, the implementation could be strengthened with additional
Table 3 Observation items with significant differences for pre- and post-test at the intervention ward in $\%$ of the total number of observations; $n=66$ at both measurements

\begin{tabular}{llll}
\hline Variable & Pre-test $[n]$ & Post-test $[n]$ & $P$ \\
\hline Inspects medication for the expiration date. & $6 \%[52]$ & $65 \%[54]$ & 0.000 \\
Inspects diluent for the expiration date. & $0 \%[52]$ & $28 \%[57]$ & 0.000 \\
Disinfects hands prior to preparation. & $15 \%[66]$ & $53 \%[66]$ & 0.000 \\
Breaks/opens ampoule with diluent using disinfectant gauze. & $15 \%[61]$ & $43 \%[65]$ & 0.000 \\
Disinfects the ampoule with medication. & $83 \%[54]$ & $98 \%[51]$ & 0.010 \\
Inspects the solution for clarity. & $5 \%[66]$ & $20 \%[66]$ & 0.007 \\
Informs the patient about administration of the drug. & $76 \%[66]$ & $94 \%[65]$ & 0.004 \\
Inspects the intravenous catheter for phlebitis & $15 \%[55]$ & $43 \%[65]$ & 0.001 \\
$\quad$ & & & \\
\hline
\end{tabular}


methods such as improved working conditions and teaching and testing of technical skills. Furthermore, it is important to consider systems approaches to reduce medication errors [24].

Observation methods for studying medication preparation and administration errors could have influenced the results. Even when all precautions are taken to minimize the effects of observation, it is still possible that the presence of a researcher may affect nurses' behavior [21].

Some nurses told the observer that they miss some steps in the total process of administration when the observer is not there. Therefore, the error rate may have been even higher in the absence of the researcher.

This implies that scores for the quality of the preparation and administration of intravenous drugs in this study are probably somewhat biased towards more positive scores. As the procedure was identical for the two groups however, this does not threaten the validity of the effects of the intervention.

Prudence is called for when generalizing the results to other departments or other hospitals. The two departments, which were involved in this study, are departments where the incidence of preparation and administration of intravenous drugs is high. Both departments were internal medicine departments where doctors and nurses often treat patients with infectious diseases. Possibly, the nurses at these departments are more aware of the importance of hygiene and infection prevention than nurses at other departments.

\section{Conclusions}

The number of errors in the preparation and administration of intravenous drugs is high. Our study identified an unobtrusive significant difference between pre-test and post-test quality of preparation and administration of these drugs after the implementation of a new protocol. Therefore, we conclude that even with limited means and investments, the quality of the preparation and administration of intravenous drugs can be improved. The protocol developed during the pre-intervention period contributes to the improvement of the quality of preparation and administration of intravenous drugs in nursing practice.

Given the limited quality of the preparation and administration process, investing more time and money in attempts at improvement seems justified. Without these attempts, the need for patient safety in everyday patient care might be insufficiently met.

Acknowledgments The authors thank the nurses from the two nursing departments of internal medicine for giving permission for observation by the researcher.

Funding No funding was received.

Conflicts of Interest There were no conflicts of interest.

Open Access This article is distributed under the terms of the Creative Commons Attribution Noncommercial License which permits any noncommercial use, distribution, and reproduction in any medium, provided the original author(s) and source are credited.

\section{Appendix}

Scores in $\%$ of all observation items per measurement and department

\begin{tabular}{|c|c|c|c|c|c|c|c|c|}
\hline \multirow[t]{2}{*}{ Variable } & \multicolumn{4}{|c|}{ 1st measurement } & \multicolumn{4}{|c|}{ 2nd measurement } \\
\hline & $\mathrm{N}$ & contr $(\%)$ & int $(\%)$ & $\mathrm{S}^{*}$ & $\mathrm{~N}$ & contr $(\%)$ & $\operatorname{int}(\%)$ & $\mathrm{S}^{*}$ \\
\hline 1. Correct drug & 132 & 100 & 100 & - & 132 & 100 & 99 & - \\
\hline 2. Correct doses & 132 & 100 & 99 & - & 132 & 100 & 100 & - \\
\hline 3. Inspects medication for the expiration date (S) & 99 & 11 & 6 & - & 103 & 8 & 65 & + \\
\hline 4. Asks colleague to double-check the prescription & 57 & 15 & 3 & - & 44 & 10 & 4 & - \\
\hline 5. Looks up preparation procedure in a handbook & 132 & 53 & 24 & + & 132 & 50 & 17 & + \\
\hline 6. Makes a calculation for the solution to be made & 4 & 100 & 100 & - & 2 & - & 50 & - \\
\hline 7. Ask colleague to double-check calculation & 4 & 0 & 67 & - & 1 & - & 100 & - \\
\hline 8. Gets the right diluent & 117 & 98 & 95 & - & 124 & 100 & 98 & - \\
\hline 9. Inspects the diluent for the expiration date (S) & 102 & 2 & 0 & - & 109 & 0 & 28 & + \\
\hline 10. Washes hands prior to preparation & 132 & 3 & 14 & - & 132 & 9 & 24 & - \\
\hline 11. Disinfects hands prior to preparation (S) & 132 & 49 & 15 & + & 132 & 38 & 53 & - \\
\hline 12. Wears gloves during preparation & 132 & 39 & 24 & - & 132 & 50 & 27 & + \\
\hline 13. Uses disinfectant gauze to break/open the ampoule (S) & 102 & 5 & 15 & - & 115 & 6 & 43 & + \\
\hline 14. Disinfects the ampoule with medication (S) & 112 & 86 & 83 & - & 111 & 87 & 98 & - \\
\hline 15. Waits for the disinfection solution to dry & 95 & 86 & 67 & - & 102 & 81 & 88 & - \\
\hline
\end{tabular}


continued

\begin{tabular}{|c|c|c|c|c|c|c|c|c|}
\hline \multirow[t]{2}{*}{ Variable } & \multicolumn{4}{|c|}{ 1st measurement } & \multicolumn{4}{|c|}{ 2nd measurement } \\
\hline & $\mathrm{N}$ & contr $(\%)$ & $\operatorname{int}(\%)$ & $\mathrm{S}^{*}$ & $\mathrm{~N}$ & $\operatorname{contr}(\%)$ & int $(\%)$ & $\mathrm{S}^{*}$ \\
\hline 16. Disinfects injection point of infusion bag & 83 & 82 & 91 & - & 68 & 90 & 78 & - \\
\hline 17. Waits for the disinfectant to dry & 71 & 76 & 53 & - & 58 & 68 & 52 & - \\
\hline 18. Uses the prescribed quantity of solvent & 119 & 98 & 98 & - & 124 & 100 & 100 & - \\
\hline 19. Uses the prescribed method of preparation & 130 & 98 & 100 & - & 131 & 100 & 100 & - \\
\hline 20. Uses the prescribed administration process & 132 & 100 & 100 & - & 132 & 100 & 100 & - \\
\hline 21. Keeps syringe, needles and/or transfer needles sterile & 127 & 98 & 92 & - & 131 & 99 & 100 & - \\
\hline 22. Takes care that the medicine completely dissolves & 106 & 96 & 93 & - & 116 & 98 & 97 & - \\
\hline 23. Mixes the acquired infusion solution by wheeling & 118 & 11 & 7 & - & 124 & 24 & 21 & - \\
\hline 24. Inspects the solution for clarity (S) & 132 & 0 & 5 & - & 132 & 0 & 20 & + \\
\hline 25. Has - at the final stage - the prescribed dose of the drug to be administered & 132 & 94 & 91 & - & 132 & 97 & 99 & - \\
\hline 26. Puts a completely filled out label on the infusion bag/syringe & 97 & 7 & 30 & - & 76 & 19 & 10 & - \\
\hline $\begin{array}{l}\text { 27. Gets } \mathrm{NaCl} 0.9 \% \text { to flush the infusion system (because of incompatibility } \\
\text { of the medicine with the infusion solution) }\end{array}$ & 30 & 86 & 96 & - & 37 & 100 & 100 & - \\
\hline 28. Keeps syringe, needles and/or transfer needles sterile & 84 & 100 & 96 & - & 76 & 100 & 100 & - \\
\hline 29. Writes his/here initials in the medication administration system & 132 & 94 & 86 & - & 130 & 97 & 97 & - \\
\hline 30. Writes the deviate time of administration in the medication administration system & 30 & 78 & 75 & - & 8 & 80 & 67 & - \\
\hline 31. Administration done by the nurse who prepared the drug & 131 & 100 & 94 & - & 129 & 97 & 100 & - \\
\hline 32. Administrates the drug before the end of sell by after preparation & 129 & 99 & 98 & - & 124 & 98 & 100 & - \\
\hline 33. Inform the patient about administration of the drug (S) & 132 & 71 & 76 & - & 129 & 72 & 94 & + \\
\hline 34. Administrates the drug to the right patient & 132 & 100 & 100 & - & 129 & 100 & 100 & - \\
\hline $\begin{array}{l}\text { 35. Inspects the IV infusion system for phlebitis and/or extravasation prior } \\
\text { to administration (S) }\end{array}$ & 116 & 10 & 15 & - & 129 & 25 & 43 & - \\
\hline 36. Arranges for new infusion needle in case of phlebitis/extravasation & 2 & - & 50 & - & 1 & 0 & - & - \\
\hline 37. Disinfects the injection point of the infusion system or the infusion needle & 93 & 83 & 96 & - & 94 & 95 & 97 & - \\
\hline 38. Waits for the disinfectant to dry & 84 & 35 & 20 & - & 90 & 37 & 32 & - \\
\hline 39. Rinses out the drip with compatible infusion solution & 33 & 100 & 83 & - & 36 & 83 & 75 & - \\
\hline 40. Uses a new coupling & 75 & 67 & 96 & + & 54 & 58 & 91 & + \\
\hline 41. Administrates the medication using the prescribed administration time & 130 & 89 & 61 & + & 128 & 80 & 66 & - \\
\hline 42. Regulates the controller according to the described rate & 85 & 95 & 87 & - & 69 & 100 & 100 & - \\
\hline 43. Keeps needles, coupling and/or drip sterile & 129 & 97 & 100 & - & 129 & 100 & 100 & - \\
\hline 44. Administers all of the solution with medicine & 113 & 88 & 96 & - & 108 & 100 & 97 & - \\
\hline 45. Rinses out the drip with compatible infusion solution & 109 & 43 & 63 & - & 89 & 56 & 76 & - \\
\hline $\begin{array}{l}\text { 46. Describes the medication administered on the patient list in case } \\
\text { of short stay or continued infusion }\end{array}$ & 96 & 82 & 78 & - & 73 & 80 & 61 & - \\
\hline 47. Stays in the patient's room after administrating the drug & 116 & 21 & 18 & - & 110 & 30 & 25 & - \\
\hline
\end{tabular}

* = observation item with significant differences for the control department and the intervention department

(S) = observation item with significant differences for pre- and post-test at the intervention department

contr $=$ control department

int $=$ intervention department

\section{References}

1. Kohn LT, Corrigan JM, Donaldson MS. To err is human: building a safer health system. Washington DC: National academy press; 2000.

2. Leape LL, Berwick DM, Bates DW. What practices will most improve safety? Evidence-based medicine meets patient safety. JAMA. 2002;288(4):501-7. doi:10.1001/jama.288.4.501.
3. Weingart SN, Wilson RM, Gibberd RW, Harrison B. Epidemiology of medical error. BMJ. 2000;320(7237):774-7. doi: 10.1136/bmj.320.7237.774.

4. Kuitunen T, Kuisma P, Hoppu K. Medication errors made by health care professionals. Analysis of the Finnish Poison Information Centre data between 2000 and 2007. Eur J Clin Pharmacol. 2008;64(8):769-74. doi:10.1007/s00228-008-0496-4. 
5. Ashcroft DM, Cooke J. Retrospective analysis of medication incidents reported using an on-line reporting system. Pharm World Sci. 2006;28(6):359-65. doi:10.1007/s11096-006-9040-8.

6. Fry MM, Dacey C. Factors contributing to incidents in medicine administration. Part 2. Br J Nurs. 2007;16(11):676-81.

7. Fry MM, Dacey C. Factors contributing to incidents in medicine administration. Part 1. Br J Nurs. 2007;16(9):556-8.

8. Bates DW, Spell N, Cullen DJ, Burdick E, Laird N, Petersen LA, et al. The costs of adverse drug events in hospitalized patients Adverse Drug Events Prevention Study Group. JAMA. 1997; 277(4):307-11. doi:10.1001/jama.277.4.307.

9. Hartley GM, Dhillon S. An observational study of the prescribing and administration of intravenous drugs in a general hospital. Int J Pharm Pract. 1998;6:38-45.

10. Ross LM, Wallace J, Paton JY. Medication errors in a pediatric teaching hospital in the UK: five years operational experience. Arch Dis Child. 2000;83(6):492-7. doi:10.1136/adc.83.6.492.

11. Taxis K, Barber N. Ethnographic study of incidence and severity of intravenous drug errors. BMJ. 2003;326(7391):684. doi: 10.1136/bmj.326.7391.684.

12. Cousins DH, Sabatier B, Begue D, Schmitt C, Hoppe-Tichy T. Medication errors in intravenous drug preparation and administration: a multicentre audit in the UK, Germany and France. Qual Saf Health Care. 2005;14(3):190-5. doi:10.1136/qshc.2003.006 676.

13. Konick-McMahan J. Full speed ahead-with caution: pushing intravenous medications. Nursing. 1996;26(6):26-31.

14. Anselmi ML, Peduzzi M, Dos Santos CB. Errors in the administration of intravenous medication in Brazilian hospitals. J Clin Nurs. 2007;16(10):1839-47. doi:10.1111/j.1365-2702.2007.01834.x.
15. Schneider PJ, Pedersen CA, Montanya KR, Curran CR, Harpe $\mathrm{SE}$, Bohenek $\mathrm{W}$, et al. Improving the safety of medication administration using an interactive CD-ROM program. Am J Health Syst Pharm. 2006;63(1):59-64. doi:10.2146/ajhp040609.

16. Greengold NL, Shane R, Schneider P, Flynn E, Elashoff J, Hoying CL, et al. The impact of dedicated medication nurses on the medication administration error rate: a randomized controlled trial. Arch Intern Med. 2003;163(19):2359-67. doi:10.1001/ archinte.163.19.2359.

17. Keen JH. Slow down. J Emerg Nurs. 1995;21(4):323-6. doi: 10.1016/S0099-1767(05)80065-0.

18. McConnel EA. Administering an I V push injection through an existing peripheral line. Nursing. 1996;26(8):24.

19. Whitman M. The push is on: delivering medications safely by i v bolus. Nursing. 1995;25(8):52-4.

20. van den Bemt PM, Fijn R, van der Voort PH, Gossen AA, Egberts TC, Brouwers JR. Frequency and determinants of drug administration errors in the intensive care unit. Crit Care Med. 2002;30(4):846-50. doi:10.1097/00003246-200204000-00022.

21. Dean B, Barber N. Validity and reliability of observational methods for studying medication administration errors. Am J Health Syst Pharm. 2001;58(1):54-9.

22. O'hare MC, Bradley AM, Gallagher T, Shields MD. Errors in administration of intravenous drugs. BMJ. 1995;310(6):1536-7.

23. Dean BS, Barber ND. A validated, reliable method of scoring the severity of medication errors. Am J Health Syst Pharm. 1999; 56(1):57-62.

24. Anderson DJ, Webster CS. A systems approach to the reduction of medication error on the hospital ward. J Adv Nurs. 2001; 35(1):34-41. doi:10.1046/j.1365-2648.2001.01820.x. 